\title{
Topological Nematic States and Non-Abelian Lattice Dislocations
}

\author{
Maissam Barkeshli and Xiao-Liang Qi \\ Department of Physics, McCullough Building, Stanford University, Stanford, California 94305-4045, USA
}

(Received 28 March 2012; published 24 August 2012)

\begin{abstract}
An exciting new prospect in condensed matter physics is the possibility of realizing fractional quantum Hall states in simple lattice models without a large external magnetic field. A fundamental question is whether qualitatively new states can be realized on the lattice as compared with ordinary fractional quantum Hall states. Here we propose new symmetry-enriched topological states, topological nematic states, which are a dramatic consequence of the interplay between the lattice translational symmetry and topological properties of these fractional Chern insulators. The topological nematic states are realized in a partially filled flat band with a Chern number $N$, which can be mapped to an $N$-layer quantum Hall system on a regular lattice. However, in the topological nematic states the lattice dislocations can act as wormholes connecting the different layers and effectively change the topology of the space. Consequently, lattice dislocations become defects with a nontrivial quantum dimension, even when the fractional quantum Hall state being realized is, by itself, Abelian. Our proposal leads to the possibility of realizing the physics of topologically ordered states on high-genus surfaces in the lab even though the sample has only the disk geometry.
\end{abstract}

DOI: 10.1103/PhysRevX.2.031013 Subject Areas: Condensed Matter Physics, Strongly Correlated Materials, Topological Insulators

\section{INTRODUCTION}

Among the most important discoveries in condensed matter physics are the integer quantum Hall and fractional quantum Hall (FQH) states [1-4], which provided the first examples of electron fractionalization in more than one dimension and paved the way for our current understanding of topological order [5-7]. Conventionally realized in two-dimensional electron gases with a strong perpendicular magnetic field, these states exhibit a bulk energy gap and topologically protected chiral edge states. The topological order of the FQH states is characterized by ground state degeneracies that depend on spatial topology [8], and fractionalized quasiparticles [4], while the quantized Hall conductance is determined by a topological invariant - the Chern number-which for a band insulator (integer quantum Hall states) can be determined by the momentum-space flux of the Berry's phase gauge field. [9].

Since Chern numbers are generic properties of any band structure, it is natural to expect that quantum Hall states can also be realized in lattice systems without an applied magnetic field $[10,11]$, and indeed such "quantum anomalous Hall" (QAH) states may be realizable experimentally $[12,13]$. Recently, numerical evidence of fractional QAH (FQAH) states-equivalent to the 1/3 Laughlin state and non-Abelian Pfaffian state-has been found in lattice models with many-body interactions and (quasi-)flat bands with Chern number $C=1$ [14-21]. A flat band with $C=1$ is similar to a Landau level where the kinetic energy

Published by the American Physical Society under the terms of the Creative Commons Attribution 3.0 License. Further distribution of this work must maintain attribution to the author(s) and the published article's title, journal citation, and DOI. is quenched and interaction effects are maximized. Recently, a systematic wave function approach was introduced that demonstrates how to associate each FQH state in a Landau level to a counterpart in a generic $C=1$ band [22].

While known $\mathrm{FQH}$ states can be realized on lattices (see also Refs. [23-26]), a fundamental question is whether new states can emerge as well. Here we show that the answer is yes. There are two key reasons why the lattice $\mathrm{FQH}$ (i.e., FQAH) systems are different: the possibility of a band with $C>1$ and the interplay of topological order with lattice symmetries $[6,27,28]$. While each Landau level has $C=1$, a single band in a lattice system can, in principle, carry an arbitrarily high Chern number. For example, a model with $C=2$ quasiflat bands has been recently proposed [29]. Here we show that there are new topologically ordered states-topological nematic states - in a partially filled band with $C>1$. These states carry a nontrivial representation of the translational symmetry and spontaneously broken lattice rotational symmetry [30]. By using the Wannier-function representation [22], a band with Chern number $C$ can be mapped to $C$ layers of Landau levels, but the $C$ layers are cyclically permuted under certain lattice translations. This leads to a dramatic interplay of these states with the lattice translational symmetry: a pair of lattice dislocations connecting different layers corresponds to a "wormhole" in the $C$-layer FQH system. The lattice dislocations effectively change the topology of the effective $C$ layer system, giving rise to topological degeneracies. Surprisingly, the topological degeneracy associated with dislocations is nontrivial even when the state itself, in the absence of dislocations, is an Abelian topological state [31-34]. Our result provides a new possibility of realizing exponentially large topological degeneracies without using a 
"genuine" non-Abelian FQH state, and effectively provides a way to experimentally observe the spatial topologydependent ground-state degeneracies of FQH states.

\section{WANNIER-FUNCTION DESCRIPTION OF CHERN INSULATORS}

It is well known that the states in a band with a nonzero Chern number cannot be written as a superposition of Wannier functions that are localized in both directions in real space. While the Chern number provides a topological obstruction to constructing such two-dimensionally localized Wannier functions, there is no obstruction to defining Wannier functions that are localized in one direction and extended in the other direction [22,35]. It was shown recently that these one-dimensional Wannier functions provide a useful basis for constructing $\mathrm{FQH}$ wave functions on the lattice, analogous to the continuum single-particle Landau gauge wave functions for Landau levels [22]. We begin by reviewing this one-dimensional Wannier-state description of two-dimensional Chern insulators.

For a band insulator with $N$ bands, the Hamiltonian is given by

$$
H=\int d^{2} \mathbf{k} c_{\mathbf{k}}^{\dagger} h(\mathbf{k}) c_{\mathbf{k}},
$$

with $h(\mathbf{k})$ a $N \times N$ matrix and the annihilation operator $c_{\mathbf{k}}$ an $N$-component vector. In this paper we will focus on the systems with only one band occupied. Denoting the occupied band by $|\mathbf{k}\rangle$, the Berry-phase gauge field is $a_{i}(\mathbf{k}) \equiv-i\left\langle\mathbf{k}\left|\partial / \partial k_{i}\right| \mathbf{k}\right\rangle$, for $i=x, y$. The one-dimensional Wannier states are defined by

$$
\left|W\left(k_{y}, n\right)\right\rangle=\int \frac{d k_{x}}{\sqrt{2 \pi}} e^{-i k_{x} n} e^{i \varphi\left(k_{x}, k_{y}\right)}|\mathbf{k}\rangle,
$$

where $[22,36]$

$$
\begin{aligned}
\varphi\left(k_{x}, k_{y}\right)= & \frac{k_{y}}{2 \pi} \int_{0}^{2 \pi} a_{y}\left(0, p_{y}\right) d p_{y}-\int_{0}^{k_{y}} a_{y}\left(0, p_{y}\right) d p_{y} \\
& +\frac{k_{x}}{2 \pi} \int_{0}^{2 \pi} a_{x}\left(p_{x}, k_{y}\right) d p_{x}-\int_{0}^{k_{x}} a_{x}\left(p_{x}, k_{y}\right) d p_{x} .
\end{aligned}
$$

We choose the phase $\varphi\left(k_{x}, k_{y}\right)$ by the condition that the states be maximally localized in the $x$ direction [37,38]. The formalism here can be generalized to a torus with finite $L_{x}, L_{y}$, in which case the Wilson line integral is replaced by a discrete summation [39].

The Wannier states form a complete basis of the occupied subspace, and each Wannier state is localized in the $x$ direction and extended in the $y$ direction. The choice of localizing the states in the $x$ direction and keeping them extended in the $y$ direction was arbitrary; we comment on other choices and generalizations in Sec. VI. In many cases, the many-body lattice FQH states constructed using these Wannier states therefore break rotational symmetry, which is why we will refer to them as topological nematic states. They will be realized in a realistic Hamiltonian only if rotational symmetry is spontaneously broken. In some cases it is possible that even though the single-particle basis states break rotational symmetry, the many-body state preserves it, as in conventional continuum $\mathrm{FQH}$ states constructed using Landau gauge basis functions. The essential property of each Wannier state is that its centerof-mass position $x_{n}\left(k_{y}\right)=\left\langle W\left(k_{y}, n\right)|\hat{x}| W\left(k_{y}, n\right)\right\rangle$, which is also the eigenvalue of the projected position operator $\hat{x}$, is determined by the Wilson loop

$$
x_{n}\left(k_{y}\right)=n-\frac{1}{2 \pi} \int_{0}^{2 \pi} a_{x}\left(p_{x}, k_{y}\right) d p_{x} .
$$

Consequently, the Chern number [9] of the band, $C_{1}=$ $\frac{1}{2 \pi} \int d k_{x} d k_{y}\left(\partial_{x} a_{y}-\partial_{y} a_{x}\right)$, is equal to the winding number of $x_{n}\left(k_{y}\right)$ when $k_{y}$ goes from 0 to $2 \pi$ :

$$
\int_{0}^{2 \pi} \frac{\partial x_{n}\left(k_{y}\right)}{\partial k_{y}} d k_{y}=C_{1} .
$$

In other words, the Wannier states satisfy the twistedboundary condition $\left|W\left(k_{y}+2 \pi, n\right)\right\rangle=\left|W\left(k_{y}, n+C_{1}\right)\right\rangle$ and correspondingly $x_{n}\left(k_{y}+2 \pi\right)=x_{n}\left(k_{y}\right)+C_{1}$. Because of such a twisted-boundary condition, the Wannier states for $C_{1}=1$ can be labeled by one parameter, $K=k_{y}+$ $2 \pi n$, and the Wannier-state basis $\left|W_{K}\right\rangle$ is in one-to-one correspondence with the Landau-level wave functions in the Landau gauge in the ordinary quantum Hall problem [22]. For $C_{1}=2$, we have $\left|W\left(k_{y}+2 \pi, n\right)\right\rangle=\left|W\left(k_{y}, n+2\right)\right\rangle$, from which it follows that the Wannier states on even and odd sites are two distinct families, as shown in Fig. 1(a). By adiabatic continuation of the momentum $k_{y}$ one
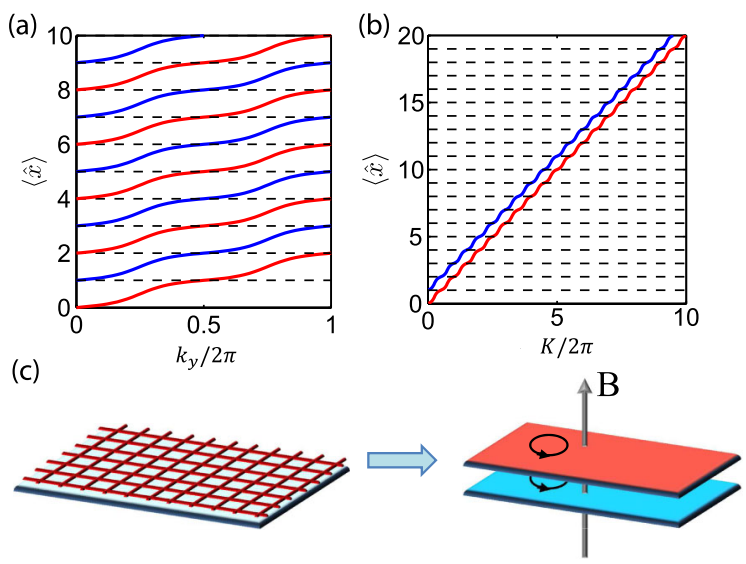

FIG. 1. (a) Each state shifts over two lattice spacings: $\langle\hat{x}\rangle \rightarrow$ $\langle\hat{x}\rangle+2$, as $k_{y} \rightarrow k_{y}+2 \pi$. Thus, there are two families of states (red and blue lines). (b) The states can be mapped to two families of states, each parametrized by a single parameter $K_{y}$. (c) Illustration of the fact that the Chern-number-2 lattice system is mapped to a bilayer quantum Hall system, with the two layers corresponding to the two families of Wannier states shown in panel (b). 
can define

$$
\begin{aligned}
& \left|W_{K=k_{y}+2 \pi n}^{1}\right\rangle=\left|W\left(k_{y}, 2 n-1\right)\right\rangle, \\
& \left|W_{K=k_{y}+2 \pi n}^{2}\right\rangle=\left|W\left(k_{y}, 2 n\right)\right\rangle,
\end{aligned}
$$

such that $\left|W_{K}^{1,2}\right\rangle$ are both continuous in the parameter $K$, and for the same $K$ they are related by a translation in the $x$ direction. Compared with the $C_{1}=1$ case, one can see that each family of states, $\left|W_{K}^{1}\right\rangle$ or $\left|W_{K}^{2}\right\rangle$, is topologically equivalent to the Wannier states of a $C_{1}=1$ Chern insulator, which is, in turn, topologically equivalent to a Landau-level quantum Hall problem. Therefore, the Wannier-state representation when $C_{1}=2$ defines a map from the $C_{1}=2$ Chern insulator to a bilayer quantum Hall problem, with the Wannier states on the even and odd sites mapped to two layers of Landau levels. In contrast to a typical bilayer quantum Hall system, the two "layers" are now related by a lattice translation. This is the key observation that leads to the topological nematic states, which we will propose below.

Multilayer $\mathrm{FQH}$ systems can exhibit a rich variety of different topological states; recently, a large classification of possibilities was developed and applied to double-layer $\mathrm{FQH}$ states [40,41]. The simplest twocomponent generalizations of the Laughlin states are the $(m n l)$ states with the wave function [42]

$$
\begin{aligned}
\Phi\left(\left\{z_{i}\right\},\left\{w_{i}\right\}\right)= & \prod_{i<j}\left(z_{i}-z_{j}\right)^{m}\left(w_{i}-w_{j}\right)^{n} \prod_{i, j}\left(z_{i}-w_{j}\right)^{l} \\
& \times \exp \left[-\sum_{i}\left(\left|z_{i}\right|^{2}+\left|w_{i}\right|^{2}\right) / 4 l_{B}^{2}\right],
\end{aligned}
$$

where $l_{B}$ is the magnetic length, $z_{i}=x_{i}+i y_{i}$ are the complex coordinates of the $i$ th particle in one layer, and similarly $w_{i}$ are the complex coordinates for the other layer. These states can be written down for the fractional Chern insulators by simply switching to the occupationnumber basis, $\Psi\left(\left\{n_{i}^{I}\right\}\right)$, where $n_{i}^{I}$ is the occupation number of the $i$ th orbital associated with the Ith layer. There are also intrinsically multilayer non-Abelian states $[40,41,43,44]$.

In the current paper, we will focus on the $(\mathrm{mml})$ states, where $m \neq l$ for states with a bulk energy gap. An important question is the following: Which states will be energetically favored in any given physically realistic Hamiltonian? In a bilayer FQH state, the $(\mathrm{mml})$ states with $l<m$ are commonly observed. This is because the interlayer repulsion $V_{\text {inter }}$ is smaller than the intralayer repulsion $V_{\text {intra. }}$. When $V_{\text {inter }} \approx V_{\text {intra, }}$, a quantum phase transition to a different many-body state is generally expected to occur [45-47]. In a flat band with Chern number $C_{1}=2$, the different layers are separated by a lattice translation. Therefore, in a model of spinless electrons with next-nearest-neighbor Hubbard interactions, it is natural that $V_{\text {inter }}$ is on the same order as, or even larger than, $V_{\text {intra }}$. Since the $(\mathrm{mml})$ states are the most stable and simplest two-component $\mathrm{FQH}$ states, we assume that they are stabilized in the ground state of some physically reasonable Hamiltonians.

\section{INTERPLAY WITH LATTICE TRANSLATIONAL SYMMETRY AND DISLOCATIONS}

A two-dimensional lattice is invariant under two independent translation operations, $T_{x}$ and $T_{y}$. Their action on the Wannier states defined in Eqs. (2) and (6) is

$$
\begin{array}{ll}
T_{x}\left|W_{K}^{1}\right\rangle=\left|W_{K}^{2}\right\rangle, & T_{x}\left|W_{K}^{2}\right\rangle=\left|W_{K+2 \pi}^{1}\right\rangle, \\
T_{y}\left|W_{K}^{a}\right\rangle=e^{i K}\left|W_{K}^{a}\right\rangle & \text { for } \quad a=1,2 .
\end{array}
$$

Thus, $T_{x}$ exchanges the two sets of Wannier states but $T_{y}$ does not. Because of the dramatically different behavior of $T_{x}$ and $T_{y}$, we see that lattice FQH states constructed using these Wannier states must break the lattice rotation symmetry. Consequently, we refer to these lattice $\mathrm{FQH}$ states as topological nematic states. In Sec. VI we will discuss generalizations which may not necessarily break lattice rotation symmetry.

Now consider the effect of dislocations [48]; these are characterized by a Burgers vector $\mathbf{b}$, which is defined as the shift of the atom position when a reference point is taken around a dislocation [51]. An $x$ dislocation with $\mathbf{b}=\hat{\mathbf{x}}$ is illustrated in Fig. 2(a). Far away from a dislocation, the lattice is locally identical to one without a dislocation, so

(a)

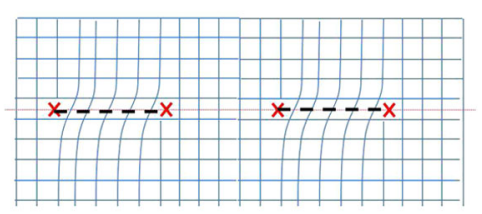

(b)

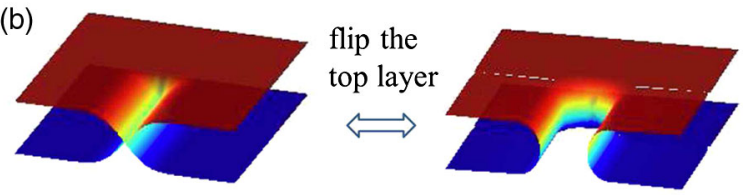

(c)
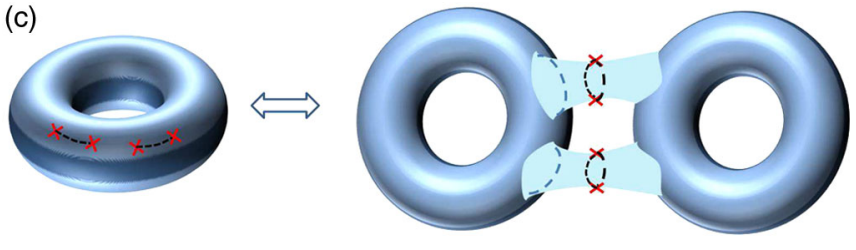

FIG. 2. (a) Illustration of an $x$ dislocation. (b) Left panel: Illustration that an $x$ dislocation leads to a branch cut around which the two effective layers are exchanged. Right panel: A reflection of the top layer maps the branch cut between a pair of dislocations into a wormhole connecting the two layers. (c) A torus with two pairs of $x$ dislocations is equivalent to two tori connected by two wormholes, which is a genus-3 surface. This picture illustrates the fact that dislocations carry nontrivial topological degeneracy. 
the dislocation is, as far as the structure of the lattice is concerned, a point defect. Now consider a bilayer $(\mathrm{mml})$ state realized on the lattice with a dislocation. As is shown in Eq. (8), the two sets of Wannier states are related by translation in the $x$ direction. Thus, when one goes around an $x$ dislocation, the two layers consisting of Wannier states $\left|W_{K}^{1}\right\rangle$ and $\left|W_{K}^{2}\right\rangle$ are exchanged. The map defined by the Wannier states, which maps the $C_{1}=2$ Chern insulator to a bilayer FQH system, maps the Chern insulator on a lattice with a pair of dislocations to a bilayer $\mathrm{FQH}$ state defined on a "Riemann surface" with a pair of branch cuts, as is illustrated in Fig. 2(b). This is the key observation that indicates that the $x$ dislocations in this system have nontrivial topological properties. By comparison, the $y$ dislocations do not exchange the two layers and thus do not correspond to a topology change in the effective bilayer description.

\section{TOPOLOGICAL DEGENERACY OF DISLOCATIONS}

Although the $(m m l)$ quantum Hall state considered is Abelian, the $x$ dislocation carries a nontrivial topological degeneracy [52]. To understand this, let us start from the simplest case of the $(\mathrm{mm} 0)$ state, which is a direct product of two Laughlin states. For such a state, the Chern insulator on a torus is mapped to two decoupled tori with a Laughlin $1 / m$ state defined on each of them, with total ground-state degeneracy of $m^{2}$. When a pair of $x$ dislocations is introduced, the two tori are connected by the branch cut. If we perform a reflection of the top layer with respect to the $x$ axis, the branch cut becomes a wormhole between the two layers, as is illustrated in Fig. 2(b). Thus, the two tori are connected, resulting in a genus 2 surface. For two pairs of dislocations, the two layers are connected by two wormholes and the whole system is topologically equivalent to a single Laughlin $1 / m$ state on a genus 3 surface, as is shown in Fig. 2(c). Thus, the ground-state degeneracy becomes $m^{3}$. In general, when there are $2 n x$ dislocations on the lattice, the space is effectively a genus $n+1$ surface and the ground-state degeneracy for $n>0$ is $m^{n+1}$. It follows that the average degree of freedom carried by each dislocation-known as the quantum dimension-is $d=\sqrt{m}$. Thus, we can see that the $x$ dislocation carries a nontrivial topological degeneracy in the same way as a non-Abelian topological quasiparticle.

The discussion above can be generalized: For the $(\mathrm{mml})$ state, $n>0$ pairs of dislocations on a torus lead to the topological degeneracy of $\left|m^{2}-l^{2}\right||m-l|^{n-1}$, as a result the quantum dimension of each dislocation is $d=$ $\sqrt{|m-l|}$ (recall $m \neq l$ for incompressible FQH states). The topological degeneracy can be computed from the bulk Chern-Simons (CS) effective theory [31]. In the following, we provide an alternative understanding of the topological degeneracy using the edge states in order to ensure that the bulk field theoretic analysis does not hide any unappreciated subtleties and to provide a deeper understanding of this phenomenon.

\section{TOPOLOGICAL DEGENERACY FROM THE EDGE-STATE PICTURE}

Here we will study in detail the topological degeneracy in the topological nematic states with dislocations by using an edge-state picture. By cutting the $\mathrm{FQH}$ state on a compact manifold along a line, one obtains a FQH state with open boundaries and gapless counterpropagating chiral edge states on the boundary. The FQH state before the cut can be obtained by "gluing" the edge states back by introducing interedge-electron tunneling [Fig. 3(a)]. The topological degeneracy comes from the fact that there are, in general, multiple degenerate minima when the electron tunneling becomes relevant [53]. In other words, the topologically degenerate ground states of the bulk topological system correspond to degenerate ground states due to spontaneous symmetry breaking in the edge theory.

The topological degeneracy of the $x$ dislocations is independent of their location, so, to compute the topological degeneracy, we may orient the $n$ pairs of $x$ dislocations along a single line. Next, we cut the system along this line to obtain a gapless conformal field theory along the cut, and then we understand the resulting ground-state degeneracy by coupling the two sides of the cut in the appropriate way.

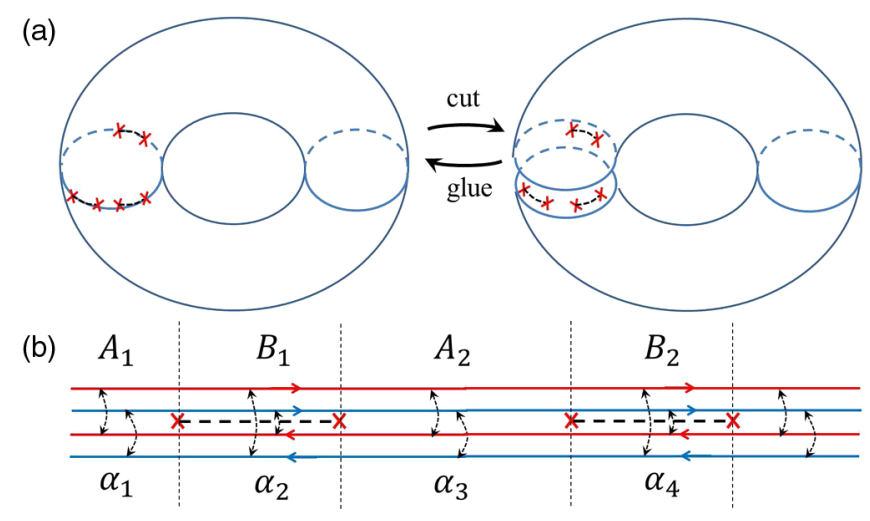

FIG. 3. Topological degeneracy seen from the edge-state perspective. (a) The dislocations are oriented along a single line, and then the system is cut along the line, yielding gapless counterpropagating edge states along the line. The original $\mathrm{FQH}$ state is obtained from gluing the system back together by turning on appropriate interedge-tunneling terms. (b) Depiction of the two branches (red and blue) of counterpropagating edge excitations. The arrows between the edge states indicate the kinds of electron-tunneling terms that are added. Away from the dislocations, in the $A$ regions, the usual electron-tunneling terms involving tunneling between the same layers, $\Psi_{e R I}^{\dagger} \Psi_{e L I}+$ H.c., are added. In the regions that include the branch cuts separating the dislocations, twisted-tunneling terms are added: $\Psi_{e R 1}^{\dagger} \Psi_{e L 2}+\Psi_{e R 2}^{\dagger} \Psi_{e L 1}+$ H.c. $\alpha_{i}$ indicate the midpoints of the $A$ or $B$ regions. 
Below, we first review the chiral edge theory of Abelian $\mathrm{FQH}$ states and how to understand their torus degeneracy from the point of view of the edge theory. Next, we show how this cut-and-glue procedure can be used to compute the ground-state degeneracy in the presence of dislocations for general two-component Abelian states.

The edge theory for an Abelian FQH state described by a generic $K$ matrix is given by the action [53,54],

$$
S_{\text {edge }}=\frac{1}{4 \pi} \int d x d t\left[K_{I J} \partial_{t} \phi_{L I} \partial_{x} \phi_{L J}-V_{I J} \partial_{x} \phi_{L I} \partial_{x} \phi_{L J}\right] \text {, }
$$

where $\phi_{L I}$ denotes left-moving chiral bosons for $I=$ $1, \ldots, \operatorname{dim} K$ [55]. Here and below, the repeated indices $I, J$ are summed. The field $\phi_{L I}$ is a compact boson field with radius $R=1$ :

$$
\phi_{L I} \sim \phi_{L I}+2 \pi
$$

Quantizing the theory in momentum space yields [6]

$$
\left[\partial_{x} \phi_{L I}(x), \phi_{L J}(y)\right]=-i 2 \pi K_{I J}^{-1} \delta(x-y) .
$$

Integrating the above equation gives

$$
\left[\phi_{L I}(x), \phi_{L J}(y)\right]=-i \pi K_{I J}^{-1} \operatorname{sgn}(x-y) .
$$

The electric charge density associated with $\phi_{L I}$ is given by

$$
\rho_{L I}=\frac{1}{2 \pi} \partial_{x} \phi_{L I},
$$

and the $I$ th electron operator is described by the vertex operator

$$
\Psi_{e L I}=e^{i K_{I J} \phi_{L J}}
$$

Note that normal ordering will be left implicit ( i.e., $e^{i K_{I J} \phi_{L J}} \equiv: e^{i K_{I J} \phi_{L J}}:$ ). If we consider the $\mathrm{FQH}$ state on a cylinder, we will have a left-moving chiral theory on one edge, and a right-moving chiral theory on the other edge. For the right-moving theory, the edge action is

$$
S_{\text {edge }}=\frac{1}{4 \pi} \int d x d t\left[-K_{I J} \partial_{t} \phi_{R I} \partial_{x} \phi_{R J}-V_{I J} \partial_{x} \phi_{R I} \partial_{x} \phi_{R J}\right] \text {, }
$$

the charge is

$$
\rho_{R I}=\frac{1}{2 \pi} \partial_{x} \phi_{R I},
$$

and the electron operator is

$$
\Psi_{e R I}=e^{-i K_{I J} \phi_{R J}} .
$$

Now if we bring the edges close together, the electrons can tunnel from one edge to the other. The electrontunneling operators are:

$$
\frac{1}{2} \sum_{I} t_{I}\left[\Psi_{e L I}^{\dagger} \Psi_{e R I}+\Psi_{e R I}^{\dagger} \Psi_{e L I}\right]=\sum_{I} t_{I} \cos \left(K_{I J} \phi_{J}\right),
$$

where

$$
\phi_{J}=\phi_{L J}+\phi_{R J}
$$

is a nonchiral boson. Note that

$$
\left[\phi_{I}(x), \phi_{J}(y)\right]=0 .
$$

The tunneling terms generate an energy gap in the edge states and lead to a set of degenerate minima. Assuming that $t_{I}<0$, the minima occur when

$$
K_{I J} \phi_{J}=2 \pi p_{I}
$$

where $p_{I}$ is an integer. That is, the minima occur when $\phi_{I}=2 \pi K_{I J}^{-1} p_{J}$. Note that since $\phi_{I} \sim \phi_{I}+2 \pi$, the degenerate states can be labeled by an integer vector $\vec{p}$, which denotes the eigenvalues of $e^{i \phi_{I}}$ :

$$
\left\langle\vec{p}\left|e^{i \phi_{I}}\right| \vec{p}\right\rangle=e^{2 \pi K_{I J}^{-1} p_{J}},
$$

for integers $p_{I}$. Consider two different vectors, $\vec{p}$ and $\vec{p}^{\prime}$. They are equivalent if taking $\phi_{I} \rightarrow \phi_{I}+2 \pi n_{I}$, for integers $n_{I}$, takes $\vec{p} \rightarrow \vec{p}+K \vec{p}=\vec{p}^{\prime}$. Thus, the ground-state degeneracy on a torus is given by the number of inequivalent integer vectors $\vec{p}$, which is determined by $|\operatorname{Det} K|$. [56] Physically, the operator $e^{i \phi_{I}}$ corresponds to a quasiparticletunneling process, where a quasiparticle from the Ith layer is annihilated at one edge, tunnels around the torus, and is created at the other edge. Thus, the different ground states can be understood as eigenstates of such a quasiparticletunneling process.

In what follows, we specialize to the case where $K$ is a $2 \times 2$ matrix,

$$
K=\left(\begin{array}{cc}
m & l \\
l & m
\end{array}\right) .
$$

On a torus, there are $|\operatorname{Det} K|=|(m-l)(m+l)|$ different ground states. From the edge theory, these can be understood as eigenstates of $e^{i\left(\phi_{1} \pm \phi_{2}\right)}$, with eigenvalue $e^{2 \pi i p_{ \pm} /(m \pm l)}$, where $p_{ \pm}$are integers. Therefore, the eigenstates can be labeled by $\left(p_{+}, p_{-}\right)$, where

$$
\left\langle\left(p_{+}, p_{-}\right)\left|e^{i\left(\phi_{1} \pm \phi_{2}\right)}\right|\left(p_{+}, p_{-}\right)\right\rangle=e^{2 \pi i p_{ \pm} /(m \pm l)} .
$$

Now suppose we have $n$ pairs of dislocations, such that translation by the Burgers vector of the dislocation exchanges the two layers. Each pair of dislocations is separated by a branch cut. Let us align all the dislocations, and denote the regions without a branch cut as $A_{i}$, and the regions with a branch cut as $B_{i}$ [Fig. 3(a)], where now $i=$ $1, \ldots, n$. Now imagine cutting the system along this line, introducing counterpropagating chiral edge states. The gapped system with the dislocations can be understood by introducing different electron-tunneling terms in the $A$ and $B$ regions [Fig. 3(b)]: 


$$
\delta \mathcal{L}_{\text {tunn }}=\frac{g}{2} \begin{cases}\Psi_{e L 1}^{\dagger} \Psi_{e R 1}+\Psi_{e L 2}^{\dagger} \Psi_{e R 2}+\text { H.c } & \text { if } x \in A_{i} \\ \Psi_{e L 1}^{\dagger} \Psi_{e R 2}+\Psi_{e L 2}^{\dagger} \Psi_{e R 1}+\text { H.c } & \text { if } x \in B_{i} .\end{cases}
$$

Introducing the variables

$$
\tilde{\phi}_{1}=\phi_{L 1}+\phi_{R 2}, \quad \tilde{\phi}_{2}=\phi_{L 2}+\phi_{R 1},
$$

we rewrite (24) as

$$
\delta \mathcal{L}_{\text {tunn }}=g \begin{cases}\sum_{I} \cos \left(K_{I J} \phi_{J}\right) & \text { if } x \in A_{i} \\ \sum_{I} \cos \left(K_{I J} \tilde{\phi}_{J}\right) & \text { if } x \in B_{i} .\end{cases}
$$

Although formally, the tunneling Hamiltonian in $A$ and $B$ regions are only different by a permutation of the two layers, there is an important difference between the two regions. Since only the electron operators are physical local operators, there is an ambiguity in the value of $\phi_{L I}$, $\phi_{R I}$. The following transformation,

$$
\begin{gathered}
\phi_{L I} \rightarrow \phi_{L I}+\frac{2 \pi n_{+}}{m+l}-(-1)^{I} \frac{2 \pi n_{-}}{m-l}, \\
\phi_{R I} \rightarrow \phi_{R I}-\frac{2 \pi n_{+}}{m+l}+(-1)^{I} \frac{2 \pi n_{-}}{m-l},
\end{gathered}
$$

with $n_{+}, n_{-} \in \mathbb{Z}$, preserves all physical operators in each layer, which are thus considered as gauge symmetries of the system. All physical operators must be gauge invariant. In the $A$ regions, the quasiparticle-tunneling operator $e^{i \phi_{I}}$, $I=1,2$ are gauge-invariant physical operators. In contrast, in the $B$ regions $e^{i \tilde{\phi}_{I}}$ are unphysical, and only the quasiparticle pair-tunneling operator $e^{i\left(\tilde{\phi}_{1}+\tilde{\phi}_{2}\right)}$ is physical. When the strength of tunneling $g$ is large enough, the edge states acquire an energy gap and all the physical quasiparticle tunneling operators $\left(e^{i \phi_{1,2}}\right.$ in $A$ regions and $e^{i \tilde{\phi}_{1}+i \tilde{\phi}_{2}}$ in $B$ regions) are pinned to one of their classical values, which are determined by minimizing the tunneling term in each region. The ground states can be written approximately as a tensor product over the degrees of freedom in the different regions:

$$
|\psi\rangle=\otimes_{i=1}^{n}\left|a_{i}\right\rangle\left|b_{i}\right\rangle
$$

where $\left|a_{i}\right\rangle$ is a state in the Hilbert space associated with $A_{i}$, and similarly for $B_{i}$. Each $A$ region contributes a degeneracy of $|\operatorname{Det} K|=\left|m^{2}-l^{2}\right|$, while each $B$ region contributes a degeneracy of $|m+l|$ coming from the eigenvalue of $\tilde{\phi}_{1}+\tilde{\phi}_{2}=\phi_{1}+\phi_{2}$. For $n$ pairs of dislocations, this counting gives the ground-state degeneracy:

$$
N_{0}=|\operatorname{Det} K|^{n}|m+l|^{n}=\left|m^{2}-l^{2}\right|^{n}|m+l|^{n} .
$$

However, there are also additional global constraints that must be satisfied. The charge $Q_{j}$ at the $j$ th dislocation is a local physical observable, which should take a fixed value in the topologically degenerate ground states, since the states with different $Q_{j}$ are physically distinguishable and are thus generically nondegenerate. These constraints are expressed as

$$
\begin{gathered}
Q_{j}=\frac{1}{2 \pi} \int_{\alpha_{j}}^{\alpha_{j+1}} \partial_{x}\left(\phi_{1}+\phi_{2}\right) d x=\frac{n_{j}}{m+l}, \\
j=1,2, \ldots, 2 n
\end{gathered}
$$

$\alpha_{2 i-1}$ and $\alpha_{2 i}$ are the midpoints of region $A_{i}$ and $B_{i}$, respectively, so that the integration region includes the $i$ th dislocation [Fig. 3(b)]. The charge is quantized in units of $\frac{1}{m+l}$, which is determined by the eigenvalues of $\phi_{1}+\phi_{2} . n_{j}=0,1,2, \ldots,|m+l|-1$. The $2 n$ charge constraints are not completely independent of each other since the total charge, $\sum_{j} Q_{j}=\frac{1}{m+l} \sum_{j} n_{j}$, must be an integer to be consistent with the charge quantization of the whole system. Therefore, for a fixed total charge $\sum_{j} Q_{j}$, the number of independent constraints (for $n>0$ ) is $2 n-1$. Each constraint reduces the number of states by a factor of $|m+l|$ since only the sector of $\phi_{1}+\phi_{2}$ is involved. Therefore, the topological degeneracy in the presence of $n>0$ pairs of dislocations is

$$
N=\frac{N_{0}}{|m+l|^{2 n-1}}=\left|\left(m^{2}-l^{2}\right)(m-l)^{n-1}\right| .
$$

\section{MORE GENERIC TOPOLOGICAL NEMATIC STATES AND EFFECTIVE TOPOLOGICAL FIELD THEORY}

As shown above, the topological degeneracy is only associated with the $x$ dislocations and not to $y$ dislocations. Such states apparently break rotational symmetry but preserve translational symmetry, which is why we name them topological nematic states. The reason for the disparity is that the FQH states are constructed using Wannier states $\left|W_{K}^{1,2}\right\rangle$ localized in the $x$ direction. If we instead define the mapping from the lattice system to the bilayer FQH system using Wannier states localized in the $y$ direction, the resulting state will have topological degeneracy associated with $y$ dislocations. These two different types of topological states can be related by redefining the Brillouin zone. More generally, we can choose the reciprocal lattice vectors to be $\mathbf{e}_{1}=(p, q)$ and $\mathbf{e}_{2}=\left(p^{\prime}, q^{\prime}\right)$ with $\left|\mathbf{e}_{1} \times \mathbf{e}_{2}\right|=$ $p q^{\prime}-q p^{\prime}=1$, and define one-dimensional Wannier states by the Fourier transform of Bloch states along the $\mathbf{e}_{1}$ direction, as shown in Fig. 4. The Wannier states obtained in this way are localized along $\mathbf{e}_{1}$ direction. For a dislocation with the Burgers vector, $\mathbf{b}=\left(b_{x}, b_{y}\right)$, around a dislocation, the translation along the $\mathbf{e}_{1}$ direction is $\mathbf{b}$. $\mathbf{e}_{1}=b_{x} p+b_{y} q$. Therefore, the exchange of two layers and topological degeneracy only occurs when $\mathbf{b} \cdot \mathbf{e}_{1}=$ $b_{x} p+b_{y} q=1 \bmod 2$. This condition classifies the topological nematic states into four types defined by $(p, q) \bmod 2=(0,0),(0,1),(1,0),(1,1)$. The topological degeneracy is associated with $x(y)$ dislocations if and only 


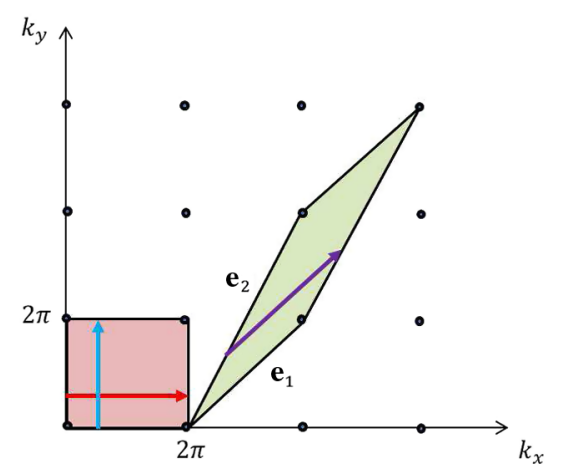

FIG. 4. Illustration of different definitions of Wannier states, which lead to different types of topological nematic states. $\mathbf{e}_{1}$ and $\mathbf{e}_{2}$ are two reciprocal vectors defining a Brillouin zone. The Wannier-state basis can be constructed by taking the Fourier transform of Bloch states along one periodic direction of the Brillouin zone, which are marked by the red, blue, and purple lines with arrows. The red, blue, and purple lines correspond to topological nematic states of the types $(1,0),(0,1)$, and $(1,1)$, respectively (see text).

if $p(q)$ is odd. Rigorously speaking, $(0,0)$ cannot be realized by any state constructed in this way, because $p q^{\prime}-q p^{\prime}=1$ requires that at least one of $p$ and one of $q$ be odd. However, one can interpret the type $(0,0)$ as the ordinary bilayer FQH state, where the two layers are not exchanged under any translation.

To describe the topological nematic states better, we have developed an effective field theory that naturally incorporates the interplay between topological properties and the lattice dislocations in this system. Note that a dislocation in a two-dimensional crystal is a defect with a long range interaction, similar to superfluid vortices. Thus, the effective field theory is different from the topological field theory studied in Ref. [31], which describes the phase with finite-energy twist defects. The proper effective field theory should satisfy the following conditions:

(1) The theory describes the dynamics of the crystal, which is characterized by the displacement field $\mathbf{u}=\left(u_{x}, u_{y}\right)$. In a $2 d$ crystal the translational symmetry $\mathbb{R} \times \mathbb{R}$ is broken to $\mathbb{Z} \times \mathbb{Z}$, such that the order parameter $\mathbf{u}$ is periodic $u_{x} \sim u_{x}+1, u_{y} \sim u_{y}+1$. Therefore, $\mathbf{u} \in U(1) \times U(1)$.

(2) The theory should reduce to a $U(1) \times U(1)$ ChernSimons theory in the absence of dislocations.

(3) When dislocations are present, the two $U(1)$ gauge groups should be exchanged when we take a reference point around a dislocation point.

A natural effective theory satisfying the conditions above can be constructed by using a $U(2)$ gauge field $a_{\mu}$, for $\mu=t, x, y$, coupled to a Higgs field $H$ of the form $H=$ $\sigma \cdot \mathbf{n} e^{i \theta(\mathbf{u})}$, where $\sigma$ are the three Pauli matrices, $\mathbf{n}$ is a real unit vector, and

$$
\theta(\mathbf{u})=\mathbf{u} \cdot \mathbf{e}_{1}
$$

is a phase factor determined by the displacement field $\mathbf{u}$. Here $\mathbf{e}_{1}$ is the reciprocal vector defining the Wannier states, as is illustrated in Fig. 4 and earlier in this section. $H$ is a traceless, unitary $2 \times 2$ matrix in the adjoint representation of $U(2)$, which transforms under a gauge transformation $H \rightarrow g^{-1} H g$, where $g$ a $U(2)$ matrix. $\mathbf{n}$ transforms as a vector under the $S U(2)$ subgroup of the $U(2)$ and $e^{i \theta}$ is gauge invariant. The effective Lagrangian has the following form:

$$
\begin{aligned}
\mathcal{L}= & \frac{1}{2}\left[\rho\left(\partial_{t} \mathbf{u}\right)^{2}-\lambda_{i j k l} \partial_{i} u_{j} \partial_{k} u_{l}\right] \\
& +\frac{m-l}{4 \pi} \epsilon^{\mu \nu \tau} \operatorname{Tr}\left[a_{\mu} \partial_{\nu} a_{\tau}+\frac{2 i}{3} a_{\mu} a_{\nu} a_{\tau}\right] \\
& +\frac{l}{4 \pi} \epsilon^{\mu \nu \tau} \operatorname{Tr}\left[a_{\mu}\right] \partial_{\nu} \operatorname{Tr}\left[a_{\tau}\right]+J \operatorname{Tr}\left[D_{\mu} H^{\dagger} D_{\mu} H\right],
\end{aligned}
$$

where the covariant derivative is $D_{\mu} H=\partial_{\mu} H+i\left[a_{\mu}, H\right]$, and $a_{\mu}=a_{\mu}^{b} \sigma^{b}$, for $b=0, x, y, z \cdot \sigma^{0}=\mathbf{1}$ is the identity matrix and $\sigma^{b}$ for $b=x, y, z$ are the Pauli matrices. The first term is the standard elasticity theory of the crystal. The Chern-Simons fields describe the topological degrees of freedom of the electrons, which are coupled with the crystal through the Higgs field $H$ [57].

We first consider a system without dislocation. For example, for $\mathbf{u}=0$ one can choose a constant Higgs field $H=\sigma^{z}$, which gives mass to two of the four components of the $U(2)$ gauge field $a$. The part of $a_{\mu}$ that remains massless is $a_{\mu}=a_{\mu}^{0} \frac{1}{2}+a_{\mu}^{3} \frac{\sigma^{z}}{2}$. Denoting $a_{\mu}^{u(d)}=\frac{1}{2}\left(a_{\mu}^{0} \pm a_{\mu}^{3}\right)$, the CS term for $a^{u}$ and $a^{d}$ reduces to

$$
\mathcal{L}_{\mathrm{CS}}=\frac{1}{4 \pi} \epsilon^{\mu \nu \tau}\left(m a_{\mu}^{u} \partial_{\nu} a_{\tau}^{u}+m a_{\mu}^{d} \partial_{\nu} a_{\tau}^{d}+2 l a_{\mu}^{u} \partial_{\nu} a_{\tau}^{d}\right),
$$

which correctly recovers the $U(1) \times U(1)$ CS theory of the ( $\mathrm{mml}$ ) state $[6,58]$, with $u$ and $d$ labeling the two layers.

Now we consider the effect of dislocations. Around a dislocation with the Burgers vector $\mathbf{b}, \theta$ changes by $\pi \mathbf{e}_{1} \cdot \mathbf{b}$. Therefore, if $e_{1} \cdot b \bmod 2=1, e^{i \theta}$ has a halfwinding configuration around the dislocation. This is allowed if, and only if, $\mathbf{n}$ also has a half-winding configuration, compensating for the minus sign from the shift of $\theta$. Such a topological defect is similar to the half vortex in a spinful $(p+i p)$ superconductor [59] but different from the $p+i p$ superconductor for which the $S U(2)$ charge is global and the $U(1)$ charge is coupled to the electromagnetic gauge field; in the current case the $S U$ (2) charge carried by the vector $\mathbf{n}$ is gauged and the $U(1)$ charge remains global. Mathematically, the space of the order parameter described by $H$ is $S^{2} \times U(1) / Z_{2}$; the $Z_{2}$ corresponds to identifying $e^{i \theta} \mathbf{n}$ with $e^{i(\theta+\pi)}(-\mathbf{n})$. $S^{2} \times U(1) / Z_{2}$ has a nontrivial fundamental group: $\pi_{1}\left[S^{2} \times U(1) / Z_{2}\right]=Z_{2}$, allowing topologically nontrivial point defects. The invariant subgroup of $U(2)$ preserving the Higgs field is $U(1) \times U(1)$, defined by the 
rotations $g=e^{i(\alpha+i \phi \mathbf{n} \cdot \sigma) / 2}$. When we adiabatically take a point around a dislocation, the vector $\mathbf{n}(\mathbf{r})$ is adiabatically rotated to $-\mathbf{n}(\mathbf{r})$, such that the two $U(1)$ 's in the invariant subgroup are exchanged. Thus the effective theory (33) reproduces the fact that the two $U(1)$ gauge fields, describing the two layers, in the $U(1) \times U(1)$ Chern-Simons theory are exchanged when a reference point is taken around a dislocation. By construction, this theory also describes the fact that dislocations have a topological degeneracy only when $e_{1} \cdot b$ is odd. Because of the gapless Goldstone modes described by the displacement field $\mathbf{u}$, the dislocations have logarithmic interaction.

\section{EVEN-ODD EFFECT AND DETECTION IN NUMERICS}

Even in the absence of dislocations, the topological nematic states exhibit unique topological characteristics that can aid in their detection in numerical simulations. To see this, below we will focus on the $(\mathrm{mml})$ topological nematic states of type $(1,0)$, defined on a lattice with periodic boundary conditions.

Let us first consider the situation shown in Fig. 5(a), where we suppose that along an entire line parallel to the $x$ direction, the hoppings have been twisted. We also suppose that the number of sites in the $x$ direction is even. Conceptually, we can imagine constructing this by taking a pair of dislocations, moving one of them around the $x$ direction of the torus, and reannihilating them. As we explain below, this change in the geometry effectively changes the topology, as in the case with dislocations. To see this, consider the $(\mathrm{mm} 0)$ states, which can be viewed as a direct product of two Laughlin $1 / m$ states. For a regular (a)

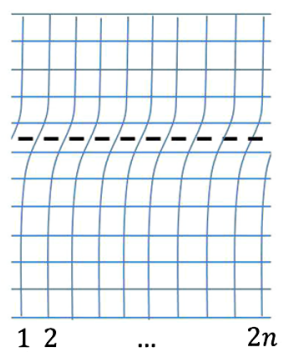

(b)

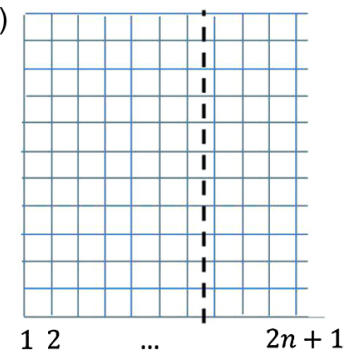

FIG. 5. Illustration of two lattice configurations to detect the topological nematic states. (a) A torus with a kink along the dashed line such that all points above the dashed line are translated by one lattice spacing in the $x$ direction relative to a regular lattice model, and with an even number of sites in the $x$ direction. (b) A regular lattice with an odd number of sites in the $x$ direction. In both cases, a periodic boundary condition is imposed on both directions. For both configurations, the $(\mathrm{mml})$ topological nematic state of type $(1,0)$ has a reduced ground-state degeneracy of $|m+l|$ instead of the degeneracy of $\left|m^{2}-l^{2}\right|$ on a torus with an even number of sites in the $x$ direction. lattice with periodic boundary conditions, the low-energy theory can be mapped onto two decoupled tori, each giving a degeneracy of $m$, for a total topological ground-state degeneracy of $\mathrm{m}^{2}$. However, the twisted hopping shown in Fig. 5(a) has the effect of gluing the two tori together into a single torus, and so we expect that the ground-state degeneracy is actually $m$. We can further see this using the edge-theory picture as presented earlier. The effect of introducing the twisted hoppings shown in Fig. 5(a) is to introduce into the edge theory the tunneling terms

$$
\delta \mathcal{L}_{\text {twisted }}=\sum_{I} \cos \left(K_{I J} \tilde{\phi}_{J}\right)
$$

with

$$
K=\left(\begin{array}{cc}
m & l \\
l & m
\end{array}\right)
$$

and $\tilde{\phi}_{J}$ defined in Eq. (25). Naively, this would give $|\operatorname{Det} K|$ different states labeled by the eigenstates of $e^{i\left(\tilde{\phi}_{1} \pm \tilde{\phi}_{2}\right)}=$ $e^{i p_{ \pm} /(m \pm l)}$, similar to the discussion in Sec. V. However, in Sec. V we discussed that $e^{i \tilde{\phi}_{I}}$ are not physical operators, and the only physical operator is the pair tunneling $e^{i \tilde{\phi}_{1}+i \tilde{\phi}_{2}}$. Therefore, we conclude that the topological degeneracy of such a twisted lattice is $|m+l|$, corresponding to the eigenvalues of $\tilde{\phi}_{1}+\tilde{\phi}_{2}$.

Alternatively, consider a regular lattice, but with an odd number of sites along the $x$ direction, as shown in Fig. 5(b). In this case, there will be a line at some point in $x$ where Wannier functions associated with different layers are adjacent. Cutting the system along this vertical line, we obtain gapless edge states, and we observe that in gluing the system back together, we again have twisted hoppings along the entire vertical line. Following a similar argument as in the last paragraph, in this case the ground-state degeneracy is $|m+l|$. This leads to a remarkable signature of the topological nematic states. For a regular lattice, when the number of sites in the $x$ direction is even, the topological degeneracy is $\left|m^{2}-l^{2}\right|$. When it is odd, the topological degeneracy is $|m+l|$. These topological degeneracies are independent of the length in the $y$ direction. Such an even-odd effect can easily be used as a numerical diagnostic to test for such topological nematic states.

\section{DOMAIN WALLS AND TRANSLATIONAL SYMMETRY-PROTECTED GAPLESS MODES}

If the Hamiltonian of a strongly interacting system that realizes the topological nematic states has a discrete lattice rotational symmetry, then the topological nematic states associated with classes $(1,0)$ or $(0,1)$ (see Sec. VI) spontaneously break the discrete rotational symmetry. In such a situation, a physically realistic system can be expected to consist of domains involving different orientations. Along the domain walls, it is possible that translational symmetry is preserved along the domain wall. As we show below, the 
(a)

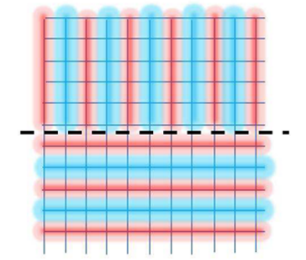

(b)

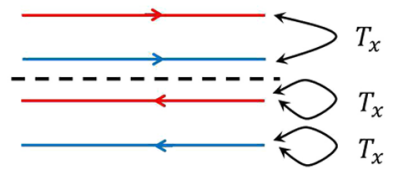

FIG. 6. (a) Illustration of the domain wall between two topological nematic states $(1,0)$ (upper half) and $(0,1)$ (lower half). (b) Illustration that the left- and right-moving edge states along the domain wall transform differently under lattice translation $T_{x}$. The right movers from the $(1,0)$ state are exchanged by $T_{x}$ while the left movers from the $(0,1)$ state are separately translation invariant.

translational symmetry along the domain wall can protect a single bosonic channel from acquiring an energy gap.

For concreteness, consider a domain wall between a $(0,1)$ and a $(1,0)$ topological nematic state, as shown in Fig. 6. Each domain on their own has two branches of chiral gapless edge states: $\phi_{L i}$ and $\phi_{R i}$, for $i=1,2$, where as before $L$ and $R$ stand for the left and right movers. Naively, one expects that these counterpropagating modes will be gapped out by interedge tunneling. However, they have different properties under translational symmetry. In particular, under a translation along the domain wall,

$$
\begin{array}{rlrl}
\phi_{L 1} & \rightarrow \phi_{L 1}, & \phi_{L 2} \rightarrow \phi_{L 2}, \\
\phi_{R 1} \rightarrow \phi_{R 2}, & \phi_{R 2} \rightarrow \phi_{R 1} .
\end{array}
$$

We see that for the translational symmetry along the domain wall to be preserved, the only allowed interedgetunneling terms must involve the symmetric combination $\cos \left(\sum_{I J} K_{I J} \phi_{J}\right)$. Therefore, either translational symmetry is preserved and the mode associated with the relative combination $\left(K_{1 J}-K_{2 J}\right) \phi_{J}$ is gapless, or translational symmetry is spontaneously broken along the domain wall. This provides a novel example of gapless edge states protected by translational symmetry.

\section{DISCUSSION AND CONCLUSIONS}

This realization that lattice dislocations carry nontrivial topological degeneracy potentially opens a number of new directions. Conceptually, we are now in need of a more general theory of topological degeneracy of dislocations and their possible braiding properties in general $\mathrm{FQH}$ states, both in bands with higher Chern numbers and in non-Abelian $\mathrm{FQH}$ states. For example, for a Chern insulator with Chern number $C_{1}=N$, the same construction maps the lattice system to a $N$-layer $\mathrm{FQH}$ state. Dislocations are "branch cuts" with degree $N$ in such a system, around which the $N$ layers are cyclically permuted. It would also be important to verify our predictions through numerical studies. The even-odd effect discussed in

Sec. VII is expected to generalize to a $\bmod N$ behavior in the topological degeneracy for $C_{1}=N$.

Note that while the dislocations studied here have nonAbelian properties, they are spatially confined excitations of the system because the energy cost of separating dislocations grows logarithmically with their distance. In other words, they are not intrinsic excitations of the electron system, but rather extrinsic defects imposed by the lattice. Nevertheless, physical materials can easily have many lattice dislocations, and if $\mathrm{FQH}$ states are realized in a material with higher-Chern-number bands, we expect that the lattice dislocations, in the limit of low dislocation density, can induce an extensive topologically stable entropy for temperatures $T \gtrsim \Delta e^{-l / \xi}$, where $\Delta$ is the energy gap of the FQH state, $\xi \propto 1 / \Delta$ is the correlation length of the gapped FQH state, and $l$ is the typical spacing between dislocations.

A further interesting, exotic possibility is to imagine deconfinement of lattice dislocations through quantum melting of the lattice. The resulting states, if realizable, are "topological liquid crystals," as the continuous translation symmetry is restored by quantum melting the lattice, while rotational symmetry is still broken. These states may correspond to the non-Abelian orbifold states that were proposed in [60] and that are described by $U(1) \times$ $U(1) \rtimes Z_{2}$ CS theory [31]. Since there is a close relation between the dislocations of the $(\mathrm{mml})$ states and the intrinsic excitations of the orbifold states, we expect that the braiding properties of the dislocations will be closely related to the braiding of non-Abelian excitations of the orbifold states. Since the dislocations are extrinsic defects, we expect that the non-Abelian part of the braiding can be well defined in general, while the overall $U(1)$ phase will be ill defined. We leave a detailed study of the braiding for future work.

In this paper, we have assumed that an $(\mathrm{mml})$ state is realized in a flat band with Chern number $C_{1}=2$. Depending on the choice of localized Wannier functions, we have found that the states may break rotational symmetry by being sensitive to either $x$ dislocations or $y$ dislocations. However, we can also construct states that are sensitive to dislocations in both directions, or to neither. The latter two possibilities do not require rotational symmetry breaking and need not have any nematic order in principle, although our current choice of many-body wave functions do break rotational symmetry. An important direction for future work is to understand better which interactions stabilize the topological nematic states considered in this work.

\section{ACKNOWLEDGMENTS}

We would like to acknowledge inspiring discussions with Alexei Kitaev and Xiao-Gang Wen and thank the Simons Center for Geometry and Physics, Stony Brook University for hospitality where these discussions took place. We 
would like to acknowledge helpful discussions with ZhengCheng Gu, Steven A. Kivelson, and Cenke Xu. This work was supported by the Simons Foundation (M. B.), the Alfred P. Sloan foundation, and the David and Lucile Packard Foundation (X.L. Q.). We thank the KITP program, Topological Insulators and Superconductors, for its hospitality while part of this work was completed. This research was supported in part by the National Science Foundation under Grant No. NSF PHY11-25915.

[1] K. v. Klitzing, G. Dorda, and M. Pepper, New Method for High-Accuracy Determination of the Fine-Structure Constant Based on Quantized Hall Resistance, Phys. Rev. Lett. 45, 494 (1980).

[2] D.C. Tsui, H. L. Stormer, and A.C. Gossard, TwoDimensional Magnetotransport in the Extreme Quantum Limit, Phys. Rev. Lett. 48, 1559 (1982).

[3] R. B. Laughlin, Quantized Hall Conductivity in Two Dimensions, Phys. Rev. B 23, 5632 (1981).

[4] R. B. Laughlin, Anomalous Quantum Hall Effect: An Incompressible Quantum Fluid with Fractionally Charged Excitations, Phys. Rev. Lett. 50, 1395 (1983).

[5] S. C. Zhang, The Chern-Simons-Landau-Ginzburg Theory of the Fractional Quantum Hall Effect, Int. J. Mod. Phys. B 6, 25 (1992).

[6] Xiao-Gang Wen, Quantum Field Theory of Many-Body Systems: From the Origin of Sound to an Origin of Light and Electrons (Oxford University Press, Oxford, 2004).

[7] Chetan Nayak, Steven H. Simon, Ady Stern, Michael Freedman, and Sankar Das Sarma, Non-Abelian Anyons and Topological Quantum Computation, Rev. Mod. Phys. 80, 1083 (2008).

[8] X. G. Wen and Q. Niu, Ground-State Degeneracy of the Fractional Quantum Hall States in the Presence of a Random Potential and on High-Genus Riemann Surfaces, Phys. Rev. B 41, 9377 (1990).

[9] D. J. Thouless, M. Kohmoto, M.P. Nightingale, and M. den Nijs, Quantized Hall Conductance in a TwoDimensional Periodic Potential, Phys. Rev. Lett. 49, 405 (1982).

[10] F. D. M. Haldane, Model for a Quantum Hall Effect without Landau Levels: Condensed-Matter Realization of the "Parity Anomaly", Phys. Rev. Lett. 61, 2015 (1988).

[11] X. L. Qi, Y.S. Wu, and S.C. Zhang, Topological Quantization of the Spin Hall Effect in Two-Dimensional Paramagnetic Semiconductors, Phys. Rev. B 74, 085308 (2006).

[12] Chao-Xing Liu, Xiao-Liang Qi, Xi Dai, Zhong Fang, and Shou-Cheng Zhang, Quantum Anomalous Hall Effect in $\mathrm{Hg}_{1-y} \mathrm{Mn}_{y} \mathrm{Te}$ Quantum Wells, Phys. Rev. Lett. 101, 146802 (2008).

[13] Rui Yu, Wei Zhang, H. J. Zhang, S. C. Zhang, Xi Dai, and Zhong Fang, Quantized Anomalous Hall Effect in Magnetic Topological Insulators, Science 329, 61 (2010).

[14] Kai Sun, Zhengcheng Gu, Hosho Katsura, and S. Das Sarma, Nearly Flatbands with Nontrivial Topology, Phys. Rev. Lett. 106, 236803 (2011).
[15] N. Regnault and B.A. Bernevig, Fractional Chern Insulator, arXiv:1105.4867.

[16] Yang-Le Wu, B. A. Bernevig, and N. Regnault, Zoology of Fractional Chern Insulators, Phys. Rev. B 85, 075116 (2012).

[17] Yi-Fei Wang, Hong Yao, Zheng-Cheng Gu, Chang-De Gong, and D. N. Sheng, Non-Abelian Quantum Hall Effect in Topological Flat Bands, Phys. Rev. Lett. 108, 126805 (2012).

[18] Titus Neupert, Luiz Santos, Claudio Chamon, and Christopher Mudry, Fractional Quantum Hall States at Zero Magnetic Field, Phys. Rev. Lett. 106, 236804 (2011).

[19] Evelyn Tang, Jia-Wei Mei, and Xiao-Gang Wen, HighTemperature Fractional Quantum Hall States, Phys. Rev. Lett. 106, 236802 (2011).

[20] D. N. Sheng, Zheng-Cheng Gu, Kai Sun, and L. Sheng, Fractional Quantum Hall Effect in the Absence of Landau Levels, Nature Commun. 2, 389 (2011).

[21] Di Xiao, Wenguang Zhu, Ying Ran, Naoto Nagaosa, and Satoshi Okamoto, Interface Engineering of Quantum Hall Effects in Digital Heterostructures of Transition-Metal Oxides, Nature Commun. 2, 596 (2011).

[22] Xiao-Liang Qi, Generic Wave-Function Description of Fractional Quantum Anomalous Hall States and Fractional Topological Insulators, Phys. Rev. Lett. 107, 126803 (2011).

[23] John McGreevy, Brian Swingle, and Ky-Anh Tran, arXiv:1109.1569.

[24] S. A. Prameswaran, R. Roy, and S. L. Sondhi, Fractional Chern Insulators and the $W_{\infty}$ Algebra, Phys. Rev. B 85, 241308 (2012).

[25] Abolhassan Vaezi, arXiv:1105.0406.

[26] Ganpathy Murthy and R. Shankar, arXiv:1108.5501.

[27] Xiao-Gang Wen, Quantum Orders and Symmetric Spin Liquids, Phys. Rev. B 65, 165113 (2002).

[28] Yuan-Ming Lu and Ying Ran, Symmetry Protected Fractional Chern Insulators and Fractional Topological Insulators, Phys. Rev. B 85, 165134 (2012).

[29] Fa Wang and Ying Ran, Nearly Flat Band with Chern Number $c=2$ on the Dice Lattice, Phys. Rev. B 84, 241103(R) (2011).

[30] S. A. Kivelson, E. Fradkin, and V.J. Emery, Electronic Liquid-Crystal Phases of a Doped Mott Insulator, Nature (London) 393, 550 (1998).

[31] Maissam Barkeshli and Xiao-Gang Wen, $u(1) \times$ $u(1) \rtimes z_{2}$ Chern-Simons Theory and $z_{4}$ Parafermion Fractional Quantum Hall States, Phys. Rev. B 81, 045323 (2010).

[32] H. Bombin, Topological Order with a Twist: Ising Anyons from an Abelian Model, Phys. Rev. Lett. 105, 030403 (2010).

[33] Alexei Kitaev and Liang Kong, Models for Gapped Boundaries and Domain Walls, arXiv:1104.5047.

[34] Alexei Kitaev, Anyons in an Exactly Solved Model and Beyond, Ann. Phys. (N.Y.) 321, 2 (2006).

[35] Sinisa Coh and David Vanderbilt, Electric Polarization in a Chern Insulator, Phys. Rev. Lett. 102, 107603 (2009).

[36] Compared to the corresponding expression in Ref. [22], we have added the first two terms in Eq. (3), which only 
depend on $k_{y}$ and fix the gauge choice between the Wannier states of different $k_{y}$.

[37] S. Kivelson, Wannier Functions in One-Dimensional Disordered Systems: Application to Fractionally Charged Solitons, Phys. Rev. B 26, 4269 (1982).

[38] Other choices besides the maximally localized one are available as well and will lead to slightly different singleparticle Wannier functions. These differences only modify non-universal properties of the many-body wave functions that we will consider and therefore are irrelevant for the considerations in this paper.

[39] Rui Yu, Xiao-Liang Qi, Andrei Bernevig, Zhong Fang, and $\mathrm{Xi}$ Dai, An Equivalent Expression of $\mathrm{Z} 2$ Topological Invariant for Band Insulators Using the Non-Abelian Berry Connection, Phys. Rev. B 84, 075119 (2011).

[40] Maissam Barkeshli and Xiao-Gang Wen, Classification of Abelian and Non-Abelian Multilayer Fractional Quantum Hall States through the Pattern of Zeros, Phys. Rev. B 82, 245301 (2010).

[41] Maissam Barkeshli and Xiao-Gang Wen, Non-Abelian Two-Component Fractional Quantum Hall States, Phys. Rev. B 82, 233301 (2010).

[42] B. I. Halperin, Theory of the Quantized Hall Conductance, Helv. Phys. Acta 56, 75 (1983).

[43] E. Ardonne, F. J. M. van Lankvelt, A. W. W. Ludwig, and K. Schoutens, Separation of Spin and Charge in Paired Spin-Singlet Quantum Hall States, Phys. Rev. B 65, 041305(R) (2002).

[44] E. Ardonne, N. Read, E. Rezayi, and K. Schoutens, NonAbelian Spin-Singlet Quantum Hall States: Wave Functions and Quasihole State Counting, Nucl. Phys. B607, 549 (2001).

[45] X.-G. Wen, Continuous Topological Phase Transitions between Clean Quantum Hall States, Phys. Rev. Lett. 84, 3950 (2000).

[46] Maissam Barkeshli and Xiao-Gang Wen, Anyon Condensation and Continuous Topological Phase Transitions in Non-Abelian Fractional Quantum Hall States, Phys. Rev. Lett. 105, 216804 (2010).

[47] Maissam Barkeshli and Xiao-Gang Wen, Bilayer Quantum Hall Phase Transitions and the Orbifold NonAbelian Fractional Quantum Hall States, Phys. Rev. B 84, 115121 (2011).

[48] Several other topological effects induced by lattice dislocations have been studied in other topological states [49,50].

[49] Vladimir Juricic, Andrej Mesaros, Robert-Jan Slager, and Jan Zaanen, Universal Probes of Two-Dimensional
Topological Insulators: Dislocation and $\pi$ Flux, Phys. Rev. Lett. 108, 106403 (2012).

[50] Ying Ran, Yi Zhang, and Ashvin Vishwanath, OneDimensional Topologically Protected Modes in Topological Insulators with Lattice Dislocations, Nature Phys. 5, 298 (2009).

[51] P.M. Chaikin and T.C. Lubensky, Principles of Condensed Matter Physics (Cambridge University Press, Cambridge, 1995).

[52] A related situation has been studied in two-component Abelian quantum Hall states [31] where $Z_{2}$-twist defects that have the effect of exchanging $\mathrm{QH}$ layers were found to be non-Abelian quasiparticles. Subsequently, $Z_{2}$-twist defects arising from dislocations in the toric code model were studied in [32], where it was also found that they are non-Abelian anyons. The current work is the first proposal where the $Z_{2}$-twist defect can be realized by a real lattice dislocation in a realistic electronic system.

[53] X.G. Wen, Chiral Luttinger Liquid and the Edge Excitations in the Fractional Quantum Hall States, Phys. Rev. B 41, 12838 (1990).

[54] X.G. Wen, Theory of the Edge States in Fractional Quantum Hall Effects, Int. J. Mod. Phys. B 6, 1711 (1992).

[55] Strictly speaking, $\phi_{L I}$ is left-moving only if the eigenvalues of $K$ are all positive. Otherwise it is either rightmoving or a combination of right- and left-moving modes. Nevertheless, we will continue to use the subscripts $L$ or $R$ even in these more general cases.

[56] X. G. Wen, Vacuum Degeneracy of Chiral Spin States in Compactified Space, Phys. Rev. B 40, 7387 (1989).

[57] We would like to note that a topological field theory in which a Higgs field induces a transition from Abelian to non-Abelian phases has been studied in E. Fradkin, C. Nayak, and K. Schoutens, Nucl. Phys. B 546, 711 (1999). This theory is different from our theory since it describes an intrinsic non-Abelian phase, but there may be a potential relation between these two theories.

[58] X.-G. Wen and A. Zee, Classification of Abelian Quantum Hall States and Matrix Formulation of Topological Fluids, Phys. Rev. B 46, 2290 (1992).

[59] Suk Bum Chung, Hendrik Bluhm, and Eun-Ah Kim, Stability of Half-Quantum Vortices in $p_{x}+i p_{y}$ Superconductors, Phys. Rev. Lett. 99, 197002 (2007).

[60] Maissam Barkeshli and Xiao-Gang Wen, Bilayer Quantum Hall Phase Transitions and the Orbifold NonAbelian Fractional Quantum Hall States, Phys. Rev. B 84, 115121 (2011). 